\title{
Enhanced Transformer Model for Data-to-Text Generation
}

\author{
Li Gong, Josep Crego, Jean Senellart \\ SYSTRAN / 5 rue Feydeau, 75002 Paris, France \\ firstname. lastnamedsystrangroup.com
}

\begin{abstract}
Neural models have recently shown significant progress on data-to-text generation tasks in which descriptive texts are generated conditioned on database records. In this work, we present a new Transformer-based data-totext generation model which learns content selection and summary generation in an endto-end fashion. We introduce two extensions to the baseline transformer model: First, we modify the latent representation of the input, which helps to significantly improve the content correctness of the output summary; Second, we include an additional learning objective that accounts for content selection modelling. In addition, we propose two data augmentation methods that succeed to further improve performance of the resulting generation models. Evaluation experiments show that our final model outperforms current state-of-theart systems as measured by different metrics: BLEU, content selection precision and content ordering. We made publicly available the transformer extension presented in this paper ${ }^{1}$.
\end{abstract}

\section{Introduction}

Data-to-text generation is an important task in natural language generation (NLG). It refers to the task of automatically producing a descriptive text from non-linguistic structured data (tables, database records, spreadsheets, etc.). Table 1 illustrates an example of data-to-text NLG, with statistics of a NBA basketball game (top) and the corresponding game summary (bottom).

Traditional approaches perform the summary generation in two separate steps: content selection ("what to say") (Duboue and McKeown, 2001, 2003) and surface realization ("how to say it") (Stent et al., 2004; Reiter et al., 2005). After the emergence of sequence-to-sequence (S2S)

\footnotetext{
${ }^{1}$ https://github.com/gongliym/ data2text-transformer
}

learning, a variety of data-to-text generation models are proposed (Lebret et al., 2016; Mei et al., 2015; Wiseman et al., 2017) and trained in an end-to-end fashion. These models are actually conditional language models which generate summaries conditioned on the latent representation of input tables. Despite producing overall fluent text, Wiseman et al. (2017) show that NLG models perform poorly on content-oriented measures.

Different from other NLG tasks (e.g., machine translation), data-to-text generation faces several additional challenges. First, data-to-text generation models have to select the content before generating text. In machine translation, the source and target sentences are semantically equivalent to each other, whereas in data-to-text generation, the model initially selects appropriate content from the input data to secondly generate fluent sentences that incorporate the selected content. Second, the training data in data-to-text generation task is often very limited. Unlike machine translation, where training data consist of translated sentence pairs, data-to-text generation models are trained from examples composed of structured data and its corresponding descriptive summary, which are much harder to produce.

In this paper, we tackle both challenges previously discussed. We introduce a new data-to-text generation model which jointly learns content selection and text generation, and we present two data augmentation methods. More precisely, we make the following contributions:

1. We adapt the Transformer (Vaswani et al., 2017) architecture by modifying the input table representation (record embedding) and introducing an additional objective function (content selection modelling).

2. We create synthetic data following two data augmentation techniques and investigate 
their impacts on different evaluation metrics.

We show that our model outperforms current state-of-the-art systems on BLEU, content selection precision and content ordering metrics.

\section{Related Work}

Automatic summary generation has been a topic of interest for a long time (Reiter and Dale, 1997; Tanaka-Ishii et al., 1998). It has interesting applications in many different domains, such as sport game summary generation (Barzilay and Lapata, 2005; Liang et al., 2009), weather-forecast generation (Reiter et al., 2005) and recipe generation (Yang et al., 2016).

Traditional data-to-text generation approaches perform the summary generation in two separate steps: content selection and surface realization. For content selection, a number of approaches were proposed to automatically select the elements of content and extract ordering constraints from an aligned corpus of input data and output summaries (Duboue and McKeown, 2001, 2003). In (Barzilay and Lapata, 2005), the content selection is treated as a collective classification problem which allows the system to capture contextual dependencies between input data items. For surface realization, Stent et al. (2004) proposed to transform the input data into an intermediary structure and then to generate natural language text from it; Reiter et al. (2005) presented a method to generate text using consistent data-to-word rules. Angeli et al. (2010) broke up the two steps into a sequence of local decisions where they used two classifiers to select content form database and another classifier to choose a suitable template to render the content.

More recently, work on this topic has focused on end-to-end generation models. Konstas and Lapata (2012) described an end-to-end generation model which jointly models content selection and surface realization. Mei et al. (2015) proposed a neural encoder-aligner-decoder model which first encodes the entire input record dataset then the aligner module performs the content selection for the decoder to generate output summary. Some other work extends the encoder-decoder model to be able to copy words directly from the input (Yang et al., 2016; Gu et al., 2016; Gulcehre et al., 2016). Wiseman et al. (2017) investigates different data-to-text generation approaches and introduces a new corpus (ROTOWIRE, see Table 1) for the data-to-text generation task along with a series of automatic measures for the contentoriented evaluation. Based on (Wiseman et al., 2017), Puduppully et al. (2019) incorporates content selection and planing mechanisms into the encoder-decoder system and improves the stateof-the-art on the RoTOWIRE dataset.

\section{Data-to-Text Generation Model}

In this section, we first formulate the data-totext generation problem and introduce our datato-text generation baseline model. Next, we detail the extensions introduced to our baseline network, namely Record Embedding and Content Selection Modelling.

\section{Problem Statement}

The objective of data-to-text generation is to generate a descriptive summary given structured data. Input of the model consists of a table of records (see Table 1, top and middle). Let $\mathbf{s}=\left\{r_{i}\right\}_{i=1}^{I}$ be a set of records, each record $r_{i}$ consists of four features:

- Entity: the name of player or team (e.g., Celtics, LeBron James)

- Type: the table header (e.g., WIN, PTS)

-Value: the value in the table (e.g., 14, Boston)

- Info: game information (e.g., H/W, V/L) which represents the team or player is Homeor Vis-team and Win- or Loss-team.

Note that there is no order relationship in $\mathbf{s}$.

The output $\mathbf{t}$ (see Table 1, bottom) is a text document which is a descriptive summary for the record set $\mathbf{s}$. Note $\mathbf{t}=t_{1} \ldots t_{J}$ with $J$ as the document length. Pairs $(\mathbf{s}, \mathbf{t})$ constitute the training data for data-to-text generation systems. Data-totext generation probability is given by:

$$
P(\mathbf{t} \mid \mathbf{s}, \theta)=\prod_{j=1}^{J} P\left(t_{j} \mid \mathbf{s}, \mathbf{t}_{<j} ; \theta\right)
$$

where $\mathbf{t}_{<j}=t_{1} \ldots t_{j-1}$ is the generated partial document and $\theta$ is the model parameters.

\section{Data-to-Text Transformer Model}

In this section, we present how we adapt the Transformer model for the data-to-text generation tasks. First, the input embedding of Transformer encoder 
is replaced by our record embedding to better incorporate the record information. Second, a new learning objective is added into our model to improve its content-oriented performance.

\subsection{Record Embedding}

The input of data-to-text model encoder is a sequence of records. Each record is a tuple of four features (Entity, Type, Value, Info). Inspired by previous work (Yang et al., 2016; Wiseman et al., 2017; Puduppully et al., 2019), we embed features into vectors, and use the concatenation of feature embeddings as the embedding of record.

$$
\mathbf{r}_{i}=\left[\mathbf{r}_{i, 1} ; \mathbf{r}_{i, 2} ; \mathbf{r}_{i, 3} ; \mathbf{r}_{i, 4}\right]
$$

where $\mathbf{r}_{i} \in \mathbb{R}^{d i m}$ is the $i$ th record embedding in the input sequence and $\mathbf{r}_{i, j} \in \mathbb{R}^{\frac{d i m}{4}}$ is the $j$ th feature embedding in $\mathbf{r}_{i}$.

Since there is no order relationship within the records, the positional embedding of the Transformer encoder is removed.

\subsection{Content Selection Modeling}

Besides record embedding, we also add a new learning objective into the Transformer model.

As presented before, we need to select the content from the input records before generating the output summary. Some records are generally important no mater the game context, such as the team name record and team score record, whereas the importance of some other records depend on the game context. For example, a player having the highest points in the game is more likely to be mentioned in the game summary. Within the Transformer architecture, the self-attention mechanism can generate the latent representation for each record by jointly conditioning on all other records in the input dataset. A binary prediction layer is added on top of the Transformer encoder output (as shown in Figure 1) to predict whether or not one record will be mentioned in the target summary.

The architecture of our data-to-text Transformer model is shown in Figure 1. As presented before, the encoder takes the record embedding as input and generates the latent representation for each record in the input sequence. The output of encoder is then used to predict the importance of each record and also serves as the context of the decoder. The decoder of our model is the same as the original Transformer model in machine translation. It predicts the next word conditioned on the encoder output and the previous tokens in the summary sequence.

In content selection modeling, the input record sequences together with its label sequences are used to optimize the encoder by minimizing the cross-entropy loss. In language generation training, the encoder and decoder are trained together to maximize the log-likelihood of the training data. The two learning objectives are trained alternatively ${ }^{2}$.

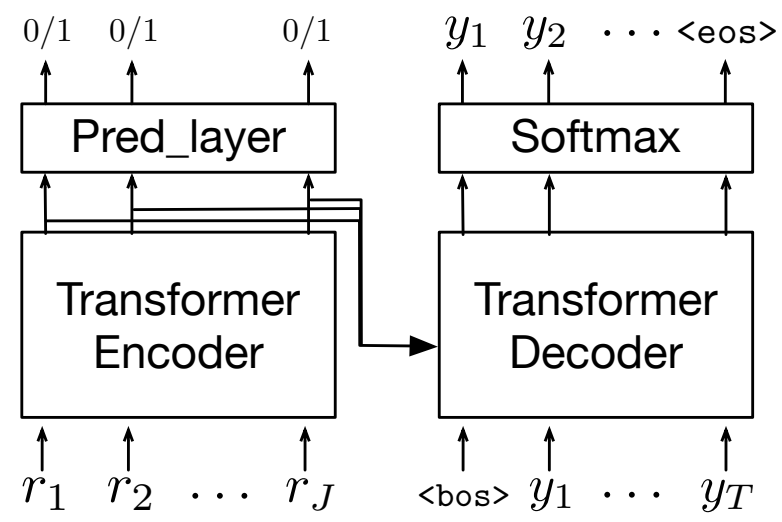

Figure 1: Model Architecture

\section{Data Augmentation Methods}

In data-to-text generation task, the model needs to not only generate fluent text, but also generate text which is coherent with the input records. Several content-oriented evaluation metrics are proposed in (Wiseman et al., 2017) to evaluate such cohesion, including the precision of record generation and the recall rate with respect to the records in gold summary.

In this section, we present two data augmentation methods: synthetic data generation and training data selection. Each of them has different impacts on the content-oriented evaluation results.

\subsection{Synthetic Data Generation}

In order to improve the cohesion between the input records and output summary, we need more data to enhance the encoder-decoder attention of the decoder. Here we introduce a method to generate synthetic training data.

We first randomly change the values of records and the changed record set $\left(\mathbf{s}^{\prime}\right)$ is then used to generate automatic summary $\left(\mathbf{t}^{\prime}\right)$ by a trained data-totext system. The synthetic data pairs $\left(\mathbf{s}^{\prime}, \mathbf{t}^{\prime}\right)$ are then used to improve such system.

\footnotetext{
${ }^{2}$ An alternative approach is joint training that achieves comparable results.
} 
This idea is inspired by the back-translation technique widely used in neural machine translation, with two important differences:

First, back-translation, typically employs monolingual human texts, which are easy found. In our case, since it is difficult to find additional structured (table) data for the same kind of game matches, we use the existing data sets and introduce variations in the values of the table records. In order to keep the data cohesion in the table, the change is constrained with the following rules:

- only numeric values are changed. Nonnumeric values such as the position of a player or the city name of a team are kept the same.

- after the change, the team scores should not violate the win/loss relation

- the changed values should stay in the normal range of its value type. It should not bigger than its maximum value or smaller than its minimum value through all games.

Our data generation technique doubles the amount of training data available for learning.

Second, another difference with the backtranslation technique is the "translation direction". In machine translation, the additional monolingual text used is found in target language, and back-translated into the source language. Thus, ensuring that the target side of the synthetic data follows the same distribution as real human texts. In our case, the target side of synthetic data is also automatically generated which is known to introduce noise in the resulting network.

\subsection{Training Data Selection}

A deficiency of data-to-text NLG systems is the poor coverage of relations produced in the generated summaries. In order to increase the coverage, a simple solution consists of learning to produce a larger number of relations. Here, we present a straightforward method to bias our model to output more relations by means of fine-tuning on the training examples containing a greater number of relations.

We use an information extraction (IE) system to extract the number of relations of each training summary. Then, we select for fine-tuning our baseline model the subset of training data in which each summary contains at least $N$ relations. In this work, we take advantage of the IE system ${ }^{3}$ provided by (Puduppully et al., 2019), and the distribution of the number of relations in the training summary is illustrated in Figure 2.

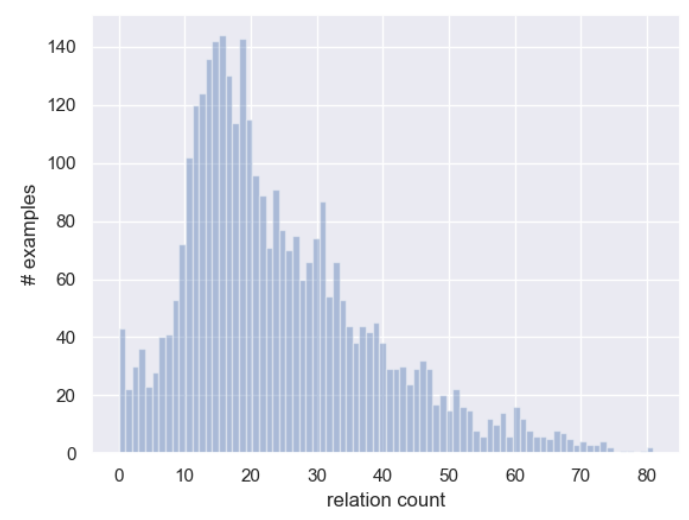

Figure 2: relation count distribution in training data.

\section{Experimental Setup}

\subsection{Data and Preprocessing}

We run the experiments with the ROTOWIRE dataset (Wiseman et al., 2017), a dataset of NBA basketball game summaries, paired with their corresponding box- and line-score tables. Table 1 illustrates an example of the dataset. In the boxscore table, each team has at most 13 players and each player is described by 23 types of values. In the line-score table, each team has 15 different types of values. In addition, the date of each game is converted into the day of the week (such as "Saturday") as an additional record. In the preprocessing step, the input box- and line-score tables are converted into a fix-length sequence of records. Each sequence contains 629 records. ${ }^{4}$ As for the associate summaries, the average length is 337 tokens, and the vocabulary size is $11.3 \mathrm{~K}$. The ROTOWIRE dataset contains 4853 summaries in total, in which 3398 summaries are for training, 727 for validation and 728 for test.

In content selection modelling, we need the labels of input records to indicate which records in the input will be mentioned in the output summary. Here we use a very simple method to gener-

\footnotetext{
${ }^{3}$ The model is publicly available at https : / / github. $\mathrm{com} / \mathrm{ratishsp/data2text-plan-py}$

${ }^{4}$ In the 629 records, 598 records are for players, 30 records for teams and 1 record for the date.
} 


\begin{tabular}{|c|c|c|c|c|c|c|c|c|c|c|c|c|c|c|c|c|c|c|c|c|}
\hline NAME & POS & MIN & PTS & FGM & FGA & FG_PCT & FG3M & FG3A & FG3_PCT & FTM & FTA & FT_PCT & OREB & DREB & REB & AST & TO & STL & BLK & $\mathrm{PF}$ \\
\hline Matt Barnes & $\mathrm{F}$ & 26 & 0 & 0 & 3 & 0 & 0 & 3 & 0 & 0 & 0 & 0 & 1 & 4 & 5 & 4 & 1 & 0 & 0 & 0 \\
\hline Blake Griffin & $\mathrm{F}$ & 34 & 24 & 10 & 17 & 59 & 0 & 0 & 0 & 4 & 5 & 80 & 4 & 2 & 6 & 8 & 4 & 1 & 0 & 3 \\
\hline DeAndre Jordan & $\mathrm{C}$ & 34 & 9 & 4 & 8 & 50 & 0 & 0 & 0 & 1 & 4 & 25 & 5 & 11 & 16 & 0 & 1 & 1 & 2 & 4 \\
\hline JJ Redick & G & 34 & 23 & 9 & 15 & 60 & 5 & 8 & 63 & 0 & 0 & 0 & 0 & 3 & 3 & 2 & 1 & 1 & 0 & 2 \\
\hline Chris Paul & G & 36 & 27 & 6 & 16 & 38 & 4 & 6 & 67 & 11 & 12 & 92 & 1 & 2 & 3 & 9 & 2 & 2 & 1 & 3 \\
\hline Glen Davis & N/A & 13 & 2 & 1 & 2 & 50 & 0 & 0 & 0 & 0 & 0 & 0 & 0 & 4 & 4 & 1 & 0 & 3 & 0 & 0 \\
\hline Jamal Crawford & N/A & 29 & 17 & 5 & 16 & 31 & 3 & 8 & 38 & 4 & 6 & 67 & 0 & 2 & 2 & 2 & 1 & 2 & 1 & 2 \\
\hline Hedo Turkoglu & N/A & 6 & 0 & 0 & 0 & 0 & 0 & 0 & 0 & 0 & 0 & 0 & 0 & 2 & 2 & 0 & 1 & 0 & 0 & 1 \\
\hline Reggie Bullock & N/A & 14 & 2 & 1 & 1 & 100 & 0 & 0 & 0 & 0 & 0 & 0 & 0 & 0 & 0 & 1 & 0 & 0 & 0 & 0 \\
\hline Jordan Farmar & N/A & 12 & 2 & 1 & 3 & 33 & 0 & 1 & 0 & 0 & 0 & 0 & 0 & 0 & 0 & 2 & 1 & 0 & 0 & 3 \\
\hline Jared Cunningha & N/A & N/A & N/A & N/A & N/A & N/A & N/A & N/A & N/A & N/A & N/A & N/A & N/A & N/A & N/A & N/A & N/A & N/A & N/A & N/A \\
\hline Chris Douglas-R & N/A & N/A & N/A & N/A & N/A & N/A & N/A & N/A & N/A & N/A & N/A & N/A & N/A & N/A & N/A & N/A & N/A & N/A & N/A & N/A \\
\hline Ekpe Udoh & N/A & N/A & N/A & N/A & N/A & N/A & N/A & N/A & N/A & N/A & N/A & N/A & N/A & N/A & N/A & N/A & N/A & N/A & N/A & N/A \\
\hline Giannis Antetok & $\mathrm{F}$ & 38 & 18 & 8 & 12 & 67 & 0 & 1 & 0 & 2 & 3 & 67 & 1 & 8 & 9 & 6 & 3 & 2 & 0 & 3 \\
\hline Johnny O’Bryant & $\mathrm{F}$ & 6 & 4 & 2 & 3 & 67 & 0 & 0 & 0 & 0 & 0 & 0 & 0 & 0 & 0 & 0 & 0 & 0 & 0 & 2 \\
\hline Larry Sanders & $\mathrm{C}$ & 26 & 10 & 5 & 6 & 83 & 0 & 0 & 0 & 0 & 2 & 0 & 2 & 5 & 7 & 3 & 0 & 1 & 1 & 5 \\
\hline O.J. Mayo & G & 23 & 3 & 1 & 6 & 17 & 0 & 2 & 0 & 1 & 3 & 33 & 0 & 1 & 1 & 3 & 2 & 0 & 0 & 4 \\
\hline Brandon Knight & G & 27 & 8 & 3 & 10 & 30 & 2 & 6 & 33 & 0 & 0 & 0 & 1 & 4 & 5 & 5 & 4 & 0 & 0 & 3 \\
\hline Jared Dudley & N/A & 30 & 16 & 7 & 12 & 58 & 2 & 4 & 50 & 0 & 0 & 0 & 2 & 6 & 8 & 3 & 3 & 2 & 0 & 2 \\
\hline Zaza Pachulia & N/A & 20 & 5 & 1 & 3 & 33 & 0 & 0 & 0 & 3 & 4 & 75 & 2 & 5 & 7 & 2 & 2 & 0 & 0 & 1 \\
\hline Jerryd Bayless & N/A & 28 & 16 & 7 & 13 & 54 & 2 & 3 & 67 & 0 & 0 & 0 & 1 & 3 & 4 & 2 & 1 & 0 & 0 & 4 \\
\hline Khris Middleton & N/A & 24 & 12 & 5 & 10 & 50 & 1 & 5 & 20 & 1 & 1 & 100 & 1 & 3 & 4 & 2 & 0 & 1 & 0 & 2 \\
\hline Kendall Marshal & N/A & 18 & 10 & 4 & 6 & 67 & 1 & 3 & 33 & 1 & 2 & 50 & 0 & 1 & 1 & 3 & 3 & 0 & 0 & 0 \\
\hline Damien Inglis & N/A & N/A & N/A & N/A & N/A & N/A & N/A & N/A & N/A & N/A & N/A & N/A & N/A & N/A & N/A & N/A & N/A & N/A & N/A & N/A \\
\hline Jabari Parker & N/A & N/A & N/A & N/A & N/A & N/A & N/A & N/A & N/A & N/A & N/A & N/A & N/A & N/A & N/A & N/A & N/A & N/A & N/A & N/A \\
\hline Nate Wolters & N/A & N/A & N/A & N/A & N/A & N/A & N/A & N/A & N/A & N/A & N/A & N/A & N/A & N/A & N/A & N/A & N/A & N/A & N/A & N/A \\
\hline TEAM-NAME & CIT & & & QTR1 & P_QT & RR2 & QTR3 & P_QTR4 & PTS $\quad \mathrm{F}$ & FG_PCT & & S3_PCT & FT_PCT & REB & AST & TOV & & VINS & LOS & SES \\
\hline Clippers & Los & Angeles & & 28 & 22 & & 32 & 24 & 106 & 46 & & 46 & 74 & 41 & 29 & 12 & & 19 & 8 & \\
\hline Bucks & Milv & aukee & & 24 & 28 & & 31 & 19 & 102 & 53 & & 33 & 53 & 46 & 29 & 18 & & 14 & 14 & \\
\hline
\end{tabular}

The Los Angeles Clippers (19-8) defeated the Milwaukee Bucks (14-14) 106-102 on Saturday. Los Angeles has won three of their last four games. Chris Paul paced the team with a game-high 27 points and nine assists. DeAndre Jordan continued his impressive work on the boards, pulling down 16 rebounds, and Blake Griffin and J.J. Redick joined Paul in scoring over 20 points. The Clippers have a tough stretch of their schedule coming up with the Spurs, Hawks, Warriors and Raptors all on this week's docket. Even with the loss, Milwaukee finished their four-game Western Conference road trip 2-2, a job well done by the developing squad. In the three games since Jabari Parker went down with a seasonending ACL injury, coach Jason Kidd has cut the umbilical cord they had on Giannis Antetokounmpo. He played over 37 minutes for the second straight game Saturday, which is ten more minutes than his season average of 27 minutes per game. Larry Sanders returned to the starting lineup after sitting out Thursday's game on a league mandated one-game suspension. Ersan Ilyasova (concussion) and John Henson (foot) remain out, and it seems Ilyasova may be closer to returning than Henson.

Table 1: An example of box-score (top), line-score (middle) and the corresponding summary (bottom) from ROTOWIRE dataset. The definition of table header could be found at https://github.com/harvardnlp/ boxscore-data

ate such labels. First, we label the entity records 5 . An entity record is labeled as 1 if its value is mentioned in the associated summary, otherwise it is labeled as 0 . Second, for each player or team mentioned in the summary, the rest of its values in the table are labeled as 1 if they occur in the same sentence in the summary.

\subsection{Evaluation metrics}

The model output is evaluated with BLEU (Papineni et al., 2002) as well as several contentoriented metrics proposed by (Wiseman et al., 2017) including three following aspects:

\footnotetext{
${ }^{5}$ Record whose Value feature is an entity (see Section 3), for example: "LeBron_James|NAME|LeBron_James|H/W". The labeling is according to the Value feature
}

- Relation Generation (RG) evaluates the number of extracted relations in automatic summaries and their correctness (precision) w.r.t the input record dataset;

- Content Selection (CS) evaluates the precision and recall rate of extracted relations in automatic summaries w.r.t that in the gold summaries;

- Content Ordering (CO) evaluates the normalized Damerau-Levenshtein Distance (Brill and Moore, 2000) between the sequence of extracted relations in automatic summaries and that in the gold summaries.

All these content-oriented metrics are based on an IE system which extracts record relations from 


\begin{tabular}{l|cc|cc|c|c}
\hline \multirow{2}{*}{ Model } & \multicolumn{2}{|c|}{ RG } & \multicolumn{2}{c|}{ CS } & CO & \multirow{2}{*}{ BLEU } \\
& $\#$ & P\% & P\% & R\% & DLD $\%$ & \\
\hline GOLD & 23.32 & 94.77 & 100 & 100 & 100 & 100 \\
\hline TEMPL & 54.29 & 99.92 & 26.61 & 59.16 & 14.42 & 8.51 \\
WS-2017 & 23.95 & 75.10 & 28.11 & 35.86 & 15.33 & 14.57 \\
NCP-2019 & 33.88 & 87.51 & 33.52 & 51.21 & 18.57 & 16.19 \\
\hline DATA-TRANS & 23.31 & 79.81 & 36.90 & 43.06 & 22.75 & 20.60 \\
+DATA_GEN & 22.59 & $\mathbf{8 2 . 4 9}$ & $\mathbf{3 9 . 4 8}$ & 42.84 & 23.32 & 19.76 \\
+DATA_SEL & $\mathbf{2 6 . 9 4}$ & 79.54 & 35.27 & $\mathbf{4 7 . 4 9}$ & 22.22 & 19.97 \\
+BOTH & 24.24 & 80.52 & 37.33 & 44.66 & 23.04 & 20.22 \\
\hline
\end{tabular}

Table 2: Automatic evaluation on RotoWIRE development set using relation generation (RG) count (\#) and precision $(\mathrm{P} \%)$, content selection $(\mathrm{CS})$ precision $(\mathrm{P} \%)$ and recall $(\mathrm{R} \%)$, content ordering $(\mathrm{CO})$ in normalized Damerau-Levenshtein distance (DLD\%), and BLEU.

summaries. For the purpose of comparison, we directly use the publicly available IE system of (Puduppully et al., 2019) to evaluate our models.

\subsection{Training Details}

In all experiments, we use our model with 1 encoder layer and 6 decoder layers, 512 hidden units (hence, the record feature embedding size is 128 , see Section 3), 8 heads, GELU activations (Hendrycks and Gimpel, 2016), a dropout rate of 0.1 and learned positional embedding for the decoder. The model is trained with the Adam optimizer (Kingma and Ba, 2014), learning rate is fixed to $10^{-4}$ and batch size is 6 . As for inference, we use beam size 4 for all experiments, and the maximum decoding length is 600 .

We implement all our models in Pytorch, and train them on 1 GTX 1080 GPU.

\section{Results}

The results of our model on the development set are summarized in Table 2. GoLD represents the evaluation result on the gold summary. The RG precision rate is $94.77 \%$, indicating that the IE system for evaluation is not perfect but has very high precision. After that, results of three contrast systems are reported, where TEMPL and WS-2017 are the updated results ${ }^{6}$ of Wiseman et al. (2017) models. TEMPL is templatebased generator model which generates a summary consisting of 8 sentences: a general description sentence about the teams playing in the game, 6 player-specific sentences and a conclusion sentence. WS-2017 reports an encoder-decoder

\footnotetext{
${ }^{6}$ Here we all use the IE system of (Puduppully et al., 2019) which is improved from the original IE system of (Wiseman et al., 2017)
}

model with conditional copy mechanism. NCP2019 is the best system configuration $(\mathrm{NCP}+\mathrm{CC})$ reported in (Puduppully et al., 2019) which is a neural content planning model enhanced with conditional copy mechanism. As for our model, results with four configurations are reported.

DATA-TRANS represents our data-to-text Transformer model (as illustrated in Figure 1) without any data augmentation. Comparing to NCP-2019, our model performs $3.4 \%$ higher on content selection precision, $4.2 \%$ higher on content ordering metric and 4.4 points higher on BLEU. Our model performs better on the $\mathrm{CO}$ metric, we attribute this improvement to that our model generates nearly the same number of relations as the gold summary which reduces the edit distance between the two sequences of relations. However, our model is $7.7 \%$ lower on RG precision. And on the CS recall rate, our model is $8.2 \%$ lower than NCP-2019. This is probably due to the fact that NCP-2019 generates much more records than our model (33.88 vs. 23.31) which could result higher coverage on the relations in gold summary.

Comparing to TEMPL and WS-2017, our model is much better on BLEU and CS precision. Our model generates nearly the same number of relations as WS-2017, but with 7.2\% higher on recall rate and $7.4 \%$ higher on $\mathrm{CO}$ metric.

By synthetic data generation (+DATA_GEN), we generate synthetic table records as described in secion 4.1. These synthetic table records are then used as input to the DATA-TRANS model to generate summaries. All training table records are used to generate synthetic data. The synthetic data is then combined with the original training data to fine-tune the DATA-TRANS model. From Table 2, we can see that the RG and CS precisions are both improved by $2.7 \%$ and $2.6 \%$ respectively. There is no significant change on others metrics. The $\mathrm{CO}$ metric is slightly improved due to higher RG and CS precisions. The CS recall rate is slightly degraded with the number of extracted relations.

By training data selection (+DATA_SEL), we select the data whose summary contains the number of relations $N>=16$ as the new training data. The result training data size is 2242 (original size: 3398). It is then used to fine-tune the DATATRANS model. As shown in Table 2, as expected, the model after fine-tuning generates more relations in the output summaries. The average num- 


\begin{tabular}{l|cc|cc|c|c}
\hline \multirow{2}{*}{ Model } & \multicolumn{2}{|c|}{ RG } & \multicolumn{2}{c|}{ CS } & CO & \multirow{2}{*}{ BLEU } \\
& $\#$ & $\mathrm{P} \%$ & $\mathrm{P} \%$ & $\mathrm{R} \%$ & DLD\% & \\
\hline TEMPL & 54.23 & 99.94 & 26.99 & 58.16 & 14.92 & 8.46 \\
WS-2017 & 23.72 & 74.80 & 29.49 & 36.18 & 15.42 & 14.19 \\
NCP-2019 & 34.28 & 87.47 & 34.18 & 51.22 & 18.58 & 16.50 \\
\hline DATA-TRANS & 24.12 & 79.17 & 36.48 & 42.74 & 22.40 & 20.16 \\
+DATA_GEN & 24.01 & $\mathbf{8 3 . 8 9}$ & $\mathbf{3 8 . 9 8}$ & 42.85 & 23.02 & 19.48 \\
+DATA_SEL & $\mathbf{2 7 . 4 7}$ & 80.70 & 35.33 & $\mathbf{4 6 . 2 5}$ & 21.87 & 20.03 \\
+BOTH & 24.80 & 81.08 & 37.10 & 43.78 & 22.51 & 20.14 \\
\hline
\end{tabular}

Table 3: Automatic evaluation on RotoWIRE test set.

\begin{tabular}{l|cc|cc|c|c}
\hline \multirow{2}{*}{ Model } & \multicolumn{2}{|c|}{ RG } & \multicolumn{2}{c|}{ CS } & CO & \multirow{2}{*}{ BLEU } \\
& $\#$ & P\% & P\% & R\% & DLD $\%$ & \\
\hline DATA-TRANS & 23.31 & 79.81 & 36.90 & 43.06 & 22.75 & 20.60 \\
-CS_OBJ & 23.37 & 72.70 & 32.67 & 41.99 & 21.14 & 20.28 \\
-REC_EMB & 18.00 & 63.14 & 32.94 & 37.71 & 21.15 & 20.24 \\
\hline
\end{tabular}

Table 4: Ablation results on RotoWIRE dev set.

ber of relations in the output summaries increases from 23.31 to 26.94. Respectively, the CS recall is increased from $43.06 \%$ to $47.49 \%$. However, the CS precision is slightly degraded by $1.6 \%$.

Finally, we combine both of the data augmentation methods $(+\mathrm{BOTH})$. Synthetic data generation improves the RG and CS precisions. Training data selection improves the CS recall rate by making the model generate more relations. To combine the two methods, we choose to fine-tune the +DATA_GEN model with the selected training data of +DATA_SEL (so this configuration is actually +DATA_GEN+DATA_SEL). As shown in Table 2, all content-oriented evaluation metrics are improved compared to DATA-TRANS but not as much as each single of the data augmentation method. This configuration is like a trade-off between the two data augmentation configurations.

Results on the test set are reported in Table 3. They follow the same pattern as those found on the development set. Our DATA-TRANS model outperforms all other contrast systems on BLEU, CS precision and content ordering metrics. The synthetic data generation method helps to improve the RG and CS precisions. The training data selection method improves the CS recall by making the model generate more relations. Combining these two data augmentation methods, all contentoriented evaluation results are improved compared to DATA-TRANS. However, there is no significant change on BLEU.

\section{Ablation Experiments}

Next we evaluate the extensions introduced in our data-to-text Transformer model (DATA-TRANS) by means of ablation experiments. This is:
- The concatenation of feature embeddings as input of the encoder presented in Section 3.1 in order to generate a better representation of the input records.

- The secondary learning objective presented in Sectioin 3.2 aiming at improving the content-oriented results.

Removing the content selection additional objective function In this configuration, we keep the same data embedding and the model architecture as the DATA-TRANS, but the model is trained without the content selection objective. The evaluation results are shown in Table 4 (-CS_OBJ). We can see that the CS precision and CS recall are degraded by $4.2 \%$ and $1 \%$ respectively. The model extracts nearly the same number of records as the baseline system, but with much lower precision. The content ordering metric is also degraded by $1.6 \%$. Surprisingly, there is no significant change on BLEU.

Removing Record Encoding In this configuration, the record encoding is removed from the DATA-TRANS model. Instead, we directly use the Value feature (see Section 3) sequence as the input. To keep model size unchanged, the dimension of embedding for the Value feature sequence is four times bigger than the original feature embedding size (see Equation 2). In addition, we also add back the positional embedding for the input sequence. Since the record sequence has a fixed length of 629 , the positional embedding could help to build a 1-to-1 mapping from the position in record sequence and the position in the real table.

The model is trained with the same data and the same configuration as DATA-TRANS. From the results in Table 4 (-REC_EMB), we can see that without record embedding all content-oriented evaluation results are degraded, especially the RG precision and CS recall. And again, the model still achieves comparable BLEU score with DATATRANS which demonstrates the effectiveness of Transformer model on language modeling.

An example output of -REC_EMB system is shown in Table 5 (left). The generation has high precision at the beginning, and many erroneous relations are generated after several sentences. Our DATA-TRANS performs much better, but we can also observe such problem. The generation has high precision at the beginning and the quality degraded after several sentences. We believe this is 
The Los Angeles Clippers (19-8) defeated the Milwaukee Bucks (14-14) 106-102 on Saturday. Milwaukee has won four straight games. They were paced by J.J. Redick's game with 23 points, five assists and five rebounds. Chris Paul had a nice game with 27 points and nine assists to go along with a double-double with nine points and nine assists. The Clippers shot 53 percent from the field and 46 percent from beyond the arc. Milwaukee will wrap up their two-game road trip in Houston against the Grizzlies on Tuesday. Milwaukee has lost four straight games. They've lost five of their last five games. Chris Paul (ankle) and Blake Griffin (knee) sat out Saturday's game. The Clippers missed their last two games with a hamstring strain. Jordan had to leave the lead the team with a foot injury but were able to return for the Clippers to action on Friday.
The Los Angeles Clippers (19-8) defeated the Milwaukee Bucks (14-14) 106-102 on Saturday. Los Angeles stopped their two-game losing streak with the win. Jamal Crawford paced the team with a game-high 17 points in 29 minutes off the bench. Crawford shot 9-of-16 from the field and 3-of-8 from downtown. He had nine assists, two rebounds and two steals in 29 minutes. Blake Griffin had 24 points, eight assists, six rebounds and one steal in 34 minutes. The Clippers will go on the road to face the Denver Nuggets on Monday. Milwaukee has lost two straight, and are now 9-2 in their last 10 games. Jabari Parker (ankle) didn't play Saturday as he recorded a double-double with 18 points and nine rebounds. Giannis Antetokounmpo (8-12 FG, 2-1 3Pt, 2-3 FT) and nine rebounds in 38 minutes off the bench. The Clippers will stay home and host the Brooklyn Nets on Monday.

Table 5: Example output from DATA-TRAnS (right) and ablation model -REC_EMB (left). The corresponding box- and line-table are given in Table 1. Text that accurately reflects a record in the associated table data is in blue, erroneous text is in red. Text in black is not contradictory to the table records and text in orange is self-contradictory within the summary.

caused by the error accumulation effect in autoregressive decoding.

Another problem we have observed, not only in Table 5 but also in other output summaries, is repetition and self-contradictory. In the left example of Table 5, it contains two sentences (in orange color) which are completely contradictory with each other. And in the right example, the sentence in orange color contains contradictory information within the sentence.

\section{Conclusions}

We presented a Transformer-based data-to-text generation model. Experimental results have shown that our two modifications on the Transformer model significantly improve the contentoriented evaluation metrics. In addition, we proposed two data augmentation methods, each of them improves different aspects of the model. Our final model outperforms current state-of-the-art system on BLEU, content selection precision and content ordering metics. And we believe it has great potential for the future work. In the next step, we would like to apply some experimental techniques of machine translation such as right-to-left decoding and system ensemble to the data-to-text generation task.

\section{References}

Gabor Angeli, Percy Liang, and Dan Klein. 2010. A simple domain-independent probabilistic approach to generation. In Proceedings of the 2010 Conference on Empirical Methods in Natural Language Processing, pages 502-512. Association for Computational Linguistics.

Regina Barzilay and Mirella Lapata. 2005. Collective content selection for concept-to-text generation. In Proceedings of the conference on Human Language Technology and Empirical Methods in Natural Language Processing, pages 331-338. Association for Computational Linguistics.

Eric Brill and Robert C Moore. 2000. An improved error model for noisy channel spelling correction. In Proceedings of the 38th Annual Meeting on Association for Computational Linguistics, pages 286-293. Association for Computational Linguistics.

Pablo A Duboue and Kathleen R McKeown. 2001. Empirically estimating order constraints for content planning in generation. In Proceedings of the 39th Annual Meeting on Association for Computational Linguistics, pages 172-179. Association for Computational Linguistics.

Pablo A Duboue and Kathleen R McKeown. 2003. Statistical acquisition of content selection rules for natural language generation. In Proceedings of the 2003 conference on Empirical methods in natural language processing, pages 121-128. Association for Computational Linguistics.

Jiatao Gu, Zhengdong Lu, Hang Li, and Victor OK Li. 2016. Incorporating copying mechanism in sequence-to-sequence learning. arXiv preprint arXiv:1603.06393. 
Caglar Gulcehre, Sungjin Ahn, Ramesh Nallapati, Bowen Zhou, and Yoshua Bengio. 2016. Pointing the unknown words. arXiv preprint arXiv:1603.08148.

Dan Hendrycks and Kevin Gimpel. 2016. Bridging nonlinearities and stochastic regularizers with gaussian error linear units.

Diederik P Kingma and Jimmy Ba. 2014. Adam: A method for stochastic optimization. arXiv preprint arXiv: 1412.6980 .

Ioannis Konstas and Mirella Lapata. 2012. Unsupervised concept-to-text generation with hypergraphs. In Proceedings of the 2012 Conference of the North American Chapter of the Association for Computational Linguistics: Human Language Technologies, pages 752-761. Association for Computational Linguistics.

Rémi Lebret, David Grangier, and Michael Auli. 2016. Neural text generation from structured data with application to the biography domain. arXiv preprint arXiv:1603.07771.

Percy Liang, Michael I Jordan, and Dan Klein. 2009. Learning semantic correspondences with less supervision. In Proceedings of the Joint Conference of the 47th Annual Meeting of the ACL and the 4th International Joint Conference on Natural Language Processing of the AFNLP: Volume 1-Volume 1, pages 91-99. Association for Computational Linguistics.

Hongyuan Mei, Mohit Bansal, and Matthew R Walter. 2015. What to talk about and how? selective generation using lstms with coarse-to-fine alignment. arXiv preprint arXiv: 1509.00838 .

Kishore Papineni, Salim Roukos, Todd Ward, and WeiJing Zhu. 2002. Bleu: a method for automatic evaluation of machine translation. In Proceedings of the 40th annual meeting on association for computational linguistics, pages 311-318. Association for Computational Linguistics.

Ratish Puduppully, Li Dong, and Mirella Lapata. 2019. Data-to-text generation with content selection and planning. In Proceedings of the AAAI Conference on Artificial Intelligence, volume 33, pages 69086915.

Ehud Reiter and Robert Dale. 1997. Building applied natural language generation systems. Natural Language Engineering, 3(1):57-87.

Ehud Reiter, Somayajulu Sripada, Jim Hunter, Jin Yu, and Ian Davy. 2005. Choosing words in computergenerated weather forecasts. Artificial Intelligence, 167(1-2):137-169.

Amanda Stent, Rashmi Prasad, and Marilyn Walker. 2004. Trainable sentence planning for complex information presentation in spoken dialog systems. In Proceedings of the 42nd annual meeting on association for computational linguistics, page 79. Association for Computational Linguistics.
Kumiko Tanaka-Ishii, Kôiti Hasida, and Itsuki Noda. 1998. Reactive content selection in the generation of real-time soccer commentary. In Proceedings of the 17th international conference on Computational linguistics-Volume 2, pages 1282-1288. Association for Computational Linguistics.

Ashish Vaswani, Noam Shazeer, Niki Parmar, Jakob Uszkoreit, Llion Jones, Aidan N Gomez, Łukasz Kaiser, and Illia Polosukhin. 2017. Attention is all you need. In Advances in neural information processing systems, pages 5998-6008.

Sam Wiseman, Stuart M Shieber, and Alexander M Rush. 2017. Challenges in data-to-document generation. arXiv preprint arXiv:1707.08052.

Zichao Yang, Phil Blunsom, Chris Dyer, and Wang Ling. 2016. Reference-aware language models. arXiv preprint arXiv:1611.01628. 